\title{
Chronic sacroiliac joint pain: fusion versus denervation as treatment options
}

Authors Bryan Ashman ${ }^{1}$, Daniel C Norvell ${ }^{2}$, Jeffrey T Hermsmeyer ${ }^{2}$

Institutions ${ }^{1}$ The Canberra Hospital, Canberra, Australia

${ }^{2}$ Spectrum Research, Inc., Tacoma, WA, USA

\section{ABSTRACT}

Study design: Systematic review

Objective: To compare the safety and effectiveness of fusion versus denervation for chronic sacroiliac joint pain after failed conservative management.

Summary of background: Methods of confirming the sacroiliac joint as a pain source have been extensively studied and reported in the literature. After confirmation of the origin of the pain by positive local anesthetic blocks, chronic sacroiliac joint pain is usually managed with a combination of medication, physical therapies, and injections. We have chosen to compare two alternative treatments for sacroiliac pain that was refractory to conservative therapies.

Methods: A systematic review of the English-language literature was undertaken for articles published between 1970 and June 2010. Electronic databases and reference lists of key articles were searched to identify studies evaluating fusion or denervation for chronic sacroiliac joint pain after failed conservative management. Studies involving only conservative treatment or traumatic onset of injury were excluded. Two independent reviewers assessed the level of evidence quality using the grading of recommendations assessment, development and evaluation (GRADE) system, and disagreements were resolved by consensus.

Results: We identified eleven articles (six fusion, five denervation) meeting our inclusion criteria. The majority of patients report satisfaction after both treatments. Both treatments reported mean improvements in pain and functional outcome. Rates of complications were higher among fusion studies (13.7\%) compared to denervation studies (7.3\%). Only fusion studies reported infections $(5.3 \%)$. No infections were reported among denervation patients. The evidence for all findings were very low to low; therefore, the relative efficacy or safety of one treatment over another cannot be established.

Conclusions: Sacroiliac joint fusion or denervation can reduce pain for many patients. Whether a true arthrodesis of the joint is achieved by percutaneous techniques is open to question and whether denervation of the joint gives durable pain relief is not clear. Further comparative studies of these two techniques may provide the answers.

This systematic review was founded by AOSpine 


\section{STUDY RATIONALE AND CONTEXT}

The sacroiliac joint as a source of low back pain has been extensively studied and reported in the literature. Pathological conditions which can affect a sacroiliac joint include degenerative and inflammatory arthritis, posttraumatic and postpartum instability, infection and neoplastic disease. Various other conditions which might cause sacroiliac joint pain include leg-length discrepancy, hip arthritis, and lumbosacral fusions for low back pain, as well as iatrogenic violation of the joint following autologous posterior iliac crest bone graft harvest. Numerous physical tests have been described to isolate the sacroiliac joint as the source of low back pain but none have proved reliable. The most accepted test for sacroiliac pain is temporary relief of the pain after injection of local anaesthetic agents into the joint under fluoroscopic $^{*}$ control. Conservative treatment of chronic sacroiliac pain has consisted of analgesic and antiinflammatory medication, physical therapies and several types of injection techniques. More invasive techniques involve fusion of the joint or denervation by ablative therapy. We have chosen to focus our review on these two techniques of long-term pain relief after failure of conservative treatment.

\section{OBJECTIVES}

To compare the effectiveness and safety of fusion versus denervation for chronic sacroiliac joint pain after failed conservative management.

\section{MATERIALS AND METHODS}

Study design: Systematic review

\section{Sampling:}

- Search: PubMed, Cochrane Collaboration Database, and National Guideline Clearinghouse Databases; bibliographies of key articles

- Dates searched: 1970-June 2010.

- Inclusion criteria: (1) chronic sacroiliac joint pain, (2) adults 18 years and older, (3) studies involving initial failed conservative treatment

- Exclusion criteria: (1) conservative treatment only, (2) unclear whether subjects had first undergone conservative treatment, (3) trauma, (4) less than five subjects per treatment, (5) less than 6-month follow-up

- Outcomes: patient satisfaction, pain, functional outcomes, wound infection, and complications (health related or surgery specific)

- Analysis: descriptive statistics pooling rates across studies

Details about methods can be found in the web appendix at www.aospine.orglebsj

\section{RESULTS}

We identified eleven articles meeting our inclusion criteria (Fig 1). Six studies evaluated fusion for sacroiliac joint pain $(\mathrm{n}=95$ patients). Five studies evaluated denervation for sacroiliac joint pain ( $\mathrm{n}=68$ patients). All studies were case series evaluating a single treatment. No cohort studies comparing one treatment to the other in the same patient population was identified, making statements regarding relative efficacy impossible. Further, follow-up times for denervation studies (6-12 months) were much shorter than fusion studies (17-69 months). All studies involved subjects who had failed other conservative management. A diagnosis of sacroiliac joint pain in all studies was confirmed by injection. Most often an injection with a solution used to reproduce exact pain pattern was followed by a local anesthetic for pain relief. Prior to their denervation treatment all patients in the denerva-

*Fluoroscopic image intensification control 
tion cohort underwent physical therapy and medical therapy. Some patients also underwent injection therapy or had previous back surgery. Many patients in the fusion cohort had previously undergone lumbar fusion and other types of back surgery.

\section{Outcomes associated with fusion versus denervation for} treatment of chronic sacroiliac pain (Table 1, Figs 2 and 3)

- The majority of fusion studies $(n=4)$ reported patient satisfaction as an outcome [1-4]. The mean rate of patient satisfaction was $57.6 \%$ (range, 18\%-100\%) among 59 subjects. Only one denervation study reported patient satisfaction. There was an $89 \%$ patient satisfaction rate out of nine subjects [5] (Table 1).

- The majority of denervation studies $(n=4)$ and two fusion studies reported pain improvement as an outcome using a visual analog or numeric rating scale to measure change from pre to posttreatment $[1,3$, 5-8]. All studies reported a mean improvement (range, 3.5-4.9 points) among subjects (Fig 2).
- Two denervation studies and one fusion study reported a change in Oswestry disability index scores from pre to posttreatment $[3,5,6]$. All studies reported a mean improvement (range, 14-18 points) (Fig 3).

Complications and infections associated with fusion and denervation for treatment of chronic sacroiliac pain (Table 1)

- The pooled infection rate among fusion studies ( $\mathrm{n}=57$ patients) was $5.3 \%$ and denervation studies ( $\mathrm{n}=68$ patients) was $0 \%$ [1, 3-10] (Table 1).

- All studies reported general health or treatment specific complications [1-11] (Table 1). The pooled complication rate (excluding infections) among fusion studies ( $\mathrm{n}=95$ patients) was $13.7 \%$ and denervation studies ( $\mathrm{n}=68$ patients) was $7.3 \%$. Fusion studies reported nonunion, pseudarthrosis and painful hardware as complications (excluding infection) while denervation studies reported transient buttock parasthesias, temporary neuritis, and numbness and itchiness of skin overlying treated sacroiliac joint.

Fig 1 Flow chart showing results of literature search

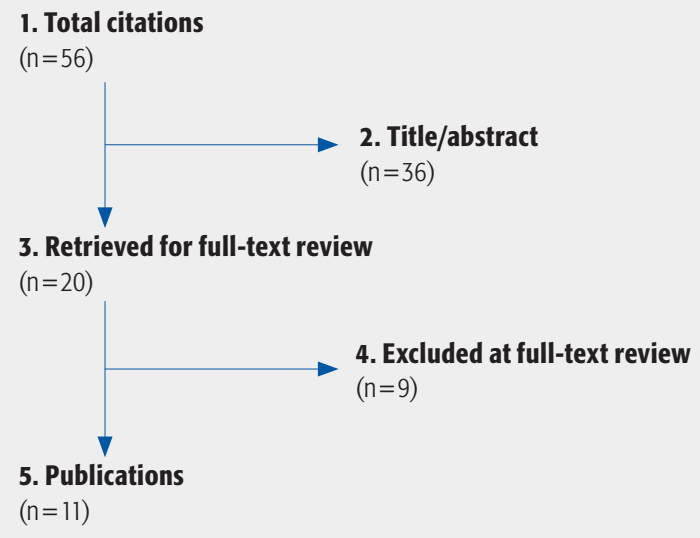

Table 1 Common outcomes of studies evaluating fusion versus denervation for treatment for sacroiliac joint pain

\begin{tabular}{lllllllll}
\hline & $\begin{array}{l}\text { Fusion } \\
\mathbf{N = 9 5}\end{array}$ & & \multicolumn{5}{c}{$\begin{array}{l}\text { Denervation } \\
\mathbf{N = 6 8}\end{array}$} \\
\hline Outcomes & Studies (n) & Patients (n) & Results (mean) & Results (range) & Studies (n) & Patients (n) & Results (mean) & Results (range) \\
\hline Patient satisfaction & 4 & 59 & $57.6 \%$ & $18 \%-100 \%$ & 1 & 9 & $89 \%$ & $89 \%$ \\
\hline Infection & 4 & 57 & $5.3 \%$ & $0 \%-11 \%$ & 5 & 68 & $0 \%$ & $0 \%$ \\
\hline All complications & 6 & 95 & $13.7 \%$ & $0 \%-41 \%$ & 5 & 68 & $7.3 \%$ & $0 \%-33 \%$ \\
\hline
\end{tabular}

*Excluding infection 
Fig 2 Mean visual analog scale or numeric rating scale improvements after fusion (blue) or denervation (grey) among all studies measuring these outcomes

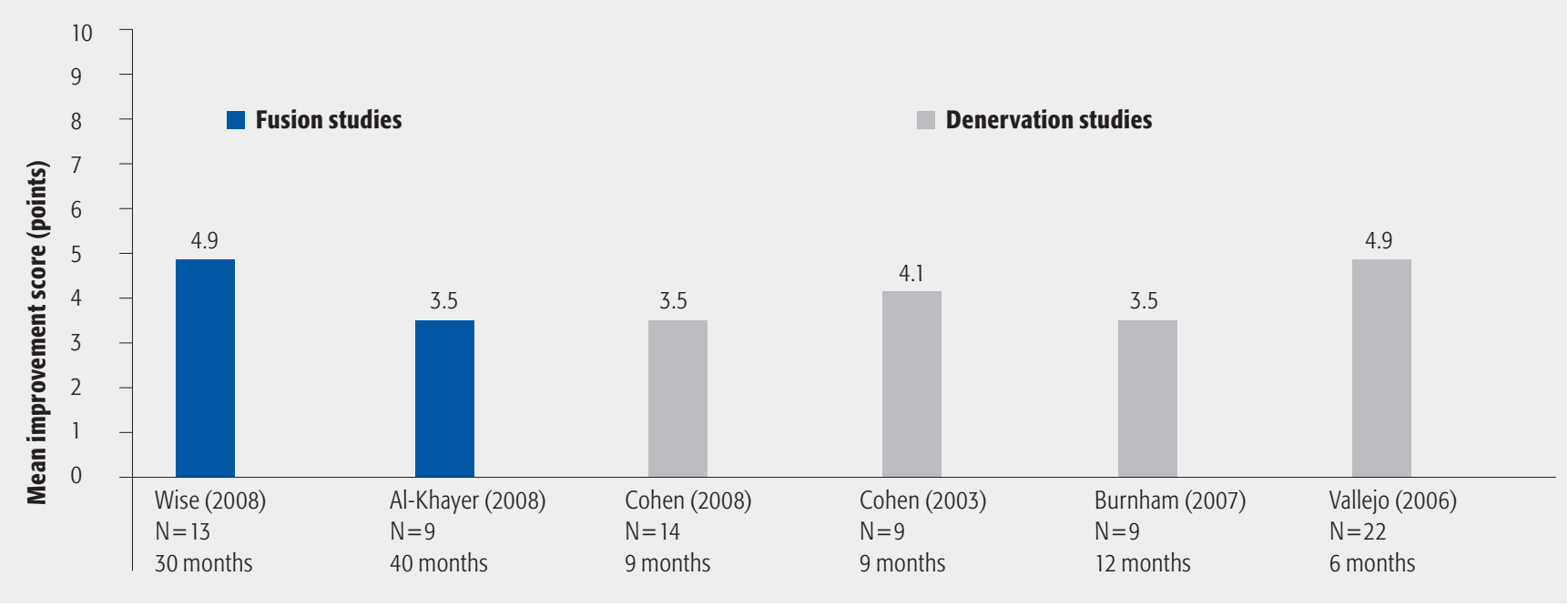

Fig 3 Oswestry disability index improvements after fusion (blue) or denervation (grey) among all studies measuring these outcomes

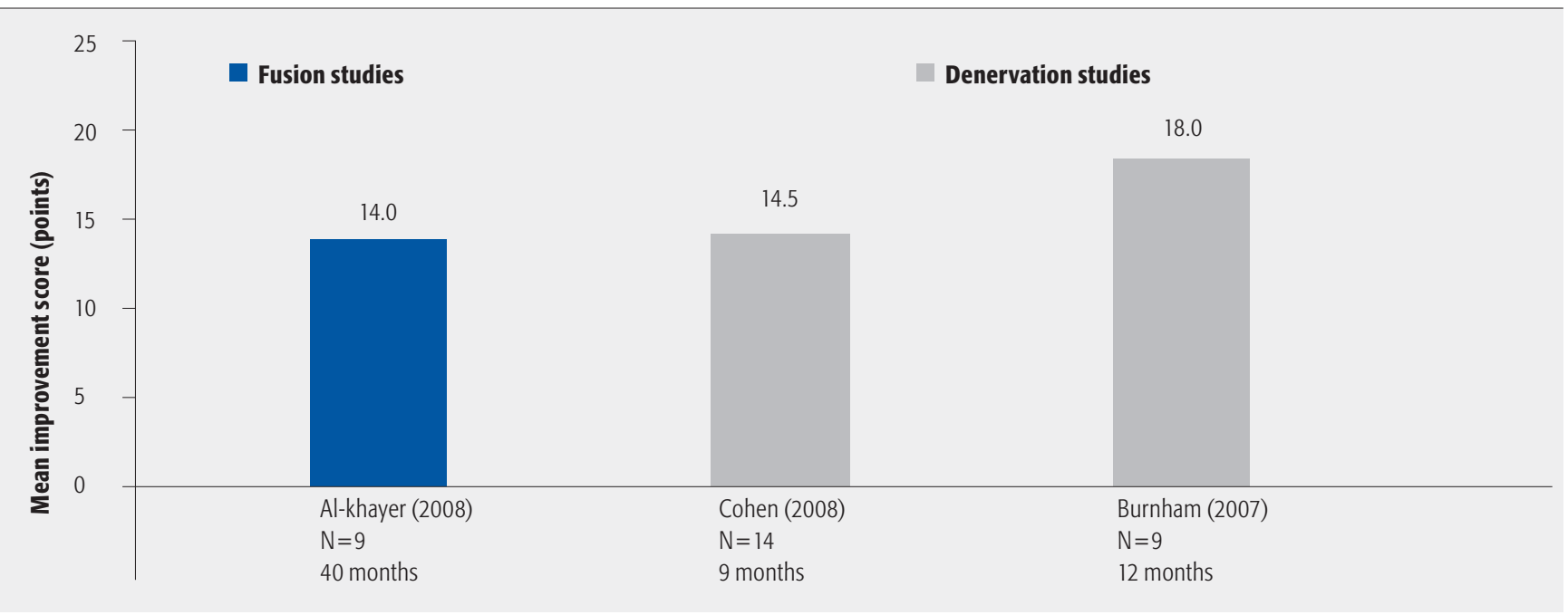


Table 2 Characteristics of studies of fusion for sacroiliac joint pain

\begin{tabular}{|c|c|c|c|c|c|c|c|}
\hline $\begin{array}{l}\text { Author } \\
\text { (year) }\end{array}$ & $\begin{array}{l}\text { Study } \\
\text { design }\end{array}$ & $\begin{array}{l}\text { Study } \\
\text { population }\end{array}$ & $\begin{array}{l}\text { Previous treatment } \\
\text { n (\%) }\end{array}$ & $\begin{array}{l}\text { Confirmed } \\
\text { diagnosis }\end{array}$ & $\begin{array}{l}\text { Fusion } \\
\text { description }\end{array}$ & $\begin{array}{l}\text { Follow-up } \\
\text { mean (range) }\end{array}$ & Outcomes \\
\hline \multirow{7}{*}{ 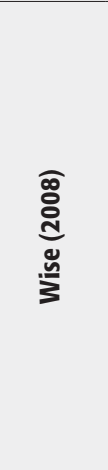 } & \multirow{7}{*}{ 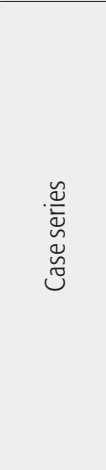 } & $\mathrm{N}=13$ patients & \multirow{7}{*}{$\begin{array}{l}\text { Failed conservative } \\
\text { therapy: } 13 \text { (100) } \\
\text { Lumbar fusion: } 8 \text { (62) } \\
\text { Open sacroiliac } \\
\text { arthrodesis on } \\
\text { contralateral side: } 1 \text { (8) }\end{array}$} & \multirow{7}{*}{$\begin{array}{l}\text { Fluoroscopically } \\
\text { guided intraarticular } \\
\text { injections of local } \\
\text { anesthetic and } \\
\text { corticosteroid } \\
\text { resulting in } 2 \text { or } \\
\text { more hours of pain } \\
\text { relief. }\end{array}$} & \multirow{7}{*}{$\begin{array}{l}\text { Percutaneously } \\
\text { inserted fusion } \\
\text { cages filled with } \\
\text { morphogenic } \\
\text { protein }\end{array}$} & \multirow{7}{*}{$\begin{array}{l}29.5 \text { months } \\
(24-35)\end{array}$} & Fusion rate: $\mathrm{n}=17 / 19(89 \%)$ \\
\hline & & Unilateral $(n=7)$ & & & & & VAS* for low back pain: improved \\
\hline & & Bilateral $(n=6)$ & & & & & average 4.9 \\
\hline & & Age: 53.1 (45-62) & & & & & VAS for leg pain: \\
\hline & & Male: $7.7 \%$ & & & & & $\begin{array}{l}\text { VAS for dyspareunia: improved } \\
\text { average } 2.6\end{array}$ \\
\hline & & & & & & & Satisfaction: $n=10 / 13(77 \%)$ \\
\hline & & & & & & & $\begin{array}{l}\text { Complications: } \\
\text { - No infections } \\
\text { - No neurovascular complications }\end{array}$ \\
\hline \multirow{9}{*}{ 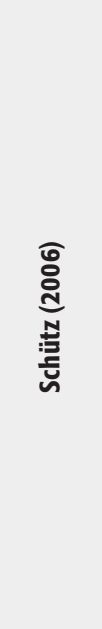 } & \multirow{9}{*}{ 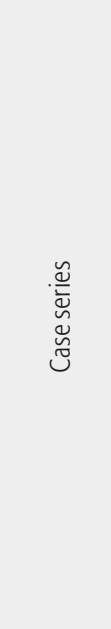 } & $\mathrm{N}=17$ patients & \multirow{9}{*}{$\begin{array}{l}\text { Failed conservative } \\
\text { therapy: } 17 \text { (100) } \\
\text { Previous lumbar surgery: } \\
10 \text { (59) } \\
\text { Previous sacroiliac joint } \\
\text { surgery: } 1 \text { (6) }\end{array}$} & \multirow{9}{*}{$\begin{array}{l}\text { Fluoroscopically } \\
\text { guided selective } \\
\text { anesthetic } \\
\text { infiltration:14 (82\%). } \\
\text { Technetium } \\
\text { bone-scan: } 5 \text { (29\%). } \\
\text { For further } \\
\text { differentiation of } \\
\text { lumbosacral and } \\
\text { sacroiliac origin of } \\
\text { pain, selective } \\
\text { immobilization of } \\
\text { lumbar, lumbosacral, } \\
\text { sacroiliac segments } \\
\text { using temporary } \\
\text { external fixator: } 4 \\
\text { (24\%) }\end{array}$} & \multirow{9}{*}{$\begin{array}{l}\text { Internal fixation } \\
\text { and decortication } \\
\text { of the sacroiliac } \\
\text { joint, using a } \\
\text { separate } \\
\text { approach to each } \\
\text { joint. Local bone } \\
\text { grafting } \\
\text { performed }\end{array}$} & \multirow{9}{*}{$\begin{array}{l}39 \text { months } \\
\left(\mathrm{NR}^{\dagger}\right)\end{array}$} & \multirow{6}{*}{$\begin{array}{l}\text { Fusion rate: } \\
\text { - Definite bony fusion: } n=6(35.3 \%) \\
\text { - Questionable fusion: } n=4(23.5 \%) \\
\text { - Nonunion with instability: } 7 \\
\text { (41.2\%) } \\
\text { Pain at follow-up: } \\
\text { - Pain free: } 1 \text { (5.8\%) } \\
\text { - Mild, intermittent: } 2(11.8 \%) \\
\text { - Marked: } 8 \text { (47.0\%) } \\
\text {-Severe, intolerable: } 6(35.3 \%)\end{array}$} \\
\hline & & Unilateral $(n=0)$ & & & & & \\
\hline & & Bilateral $(n=17)$ & & & & & \\
\hline & & Age: $43.2(22-76)$ & & & & & \\
\hline & & Male: $29.4 \%$ & & & & & \\
\hline & & & & & & & \\
\hline & & & & & & & Further surgery: $n=11$ (64.7\%) \\
\hline & & & & & & & Satisfaction: $n=3(17.6 \%)$ \\
\hline & & & & & & & Complications: NR \\
\hline \multirow{8}{*}{ 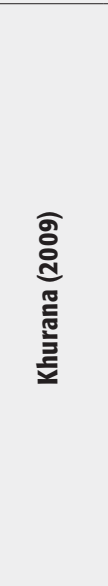 } & \multirow{8}{*}{ 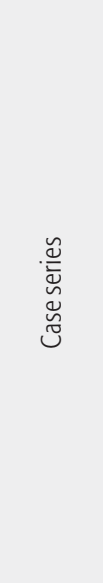 } & $\mathrm{N}=15$ patients & \multirow{8}{*}{$\begin{array}{l}\text { Previous spinal surgery: } \\
6(40)\end{array}$} & \multirow{8}{*}{$\begin{array}{l}\text { Injection of local } \\
\text { anesthetic and } \\
\text { steroid under image } \\
\text { intensifier control }\end{array}$} & \multirow{8}{*}{$\begin{array}{l}\text { Percutaneously } \\
\text { inserted hollow } \\
\text { modular } \\
\text { anchorage screws } \\
\text { filled with } \\
\text { demineralized } \\
\text { bone matrix }\end{array}$} & \multirow{8}{*}{$\begin{array}{l}17 \text { months } \\
(9-39)\end{array}$} & Fusion rate: $n=15 / 15(100 \%)$ \\
\hline & & Unilateral $(n=11)$ & & & & & SF-36 physical function: improved \\
\hline & & Bilateral $(n=4)$ & & & & & from 37 (23-51) to 80 (67-92) \\
\hline & & Age: 48.7 (37-62) & & & & & $\begin{array}{l}\text { SF-36 general health: improved } \\
\text { from } 53 \text { (34-73) to } 86 \text { (70-98) }\end{array}$ \\
\hline & & Male: $26.7 \%$ & & & & & $\begin{array}{l}\text { Majeed score: improved from } 37 \\
(18-54) \text { to } 79 \text { (62-96) }\end{array}$ \\
\hline & & & & & & & $\begin{array}{l}\text { Majeed score excellent results: } \\
13(86.7)\end{array}$ \\
\hline & & & & & & & Further surgery: $n=0(0)$ \\
\hline & & & & & & & $\begin{array}{l}\text { Complications: } \\
\text { - No infections } \\
\text { - No neurological complications } \\
\text { - No cases of implant failure }\end{array}$ \\
\hline
\end{tabular}

${ }^{*}$ VAS $=$ visual analog scale

${ }^{\dagger} \mathrm{NR}=$ not reported 


\begin{tabular}{|c|c|c|c|c|c|c|c|}
\hline $\begin{array}{l}\text { Author } \\
\text { (year) }\end{array}$ & $\begin{array}{l}\text { Study } \\
\text { design }\end{array}$ & $\begin{array}{l}\text { Study } \\
\text { population }\end{array}$ & $\begin{array}{l}\text { Previous treatment } \\
\text { n (\%) }\end{array}$ & $\begin{array}{l}\text { Confirmed } \\
\text { diagnosis }\end{array}$ & $\begin{array}{l}\text { Fusion } \\
\text { description }\end{array}$ & $\begin{array}{l}\text { Follow-up } \\
\text { mean (range) }\end{array}$ & Outcomes \\
\hline \multirow{7}{*}{ 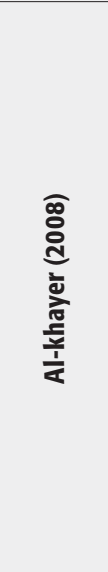 } & \multirow{7}{*}{ 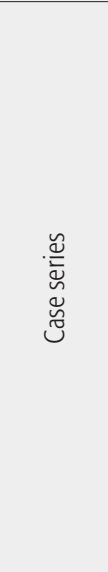 } & $\mathrm{N}=9$ patients & \multirow{7}{*}{$\begin{array}{l}\text { Failed rigorous } \\
\text { conservative therapy: } 9 \\
\text { (100) }\end{array}$} & \multirow{7}{*}{$\begin{array}{l}\text { Temporary pain } \\
\text { relief with sacroiliac } \\
\text { joint block }\end{array}$} & \multirow{7}{*}{$\begin{array}{l}\text { Percutaneously } \\
\text { inserted hollow } \\
\text { modular } \\
\text { anchorage screws } \\
\text { filled with } \\
\text { demineralized } \\
\text { bone matrix }\end{array}$} & \multirow{7}{*}{$\begin{array}{l}40 \text { months } \\
\text { (24-70) }\end{array}$} & Fusion rate: $n=9 / 9(100 \%)$ \\
\hline & & Unilateral $(n=6)$ & & & & & Owestry disability index: improved \\
\hline & & Bilateral $(n=3)$ & & & & & from 59 (34-70) to 45 (28-60) \\
\hline & & Age: $42.4(35-56)$ & & & & & $\begin{array}{l}\text { VAS for low back pain: improved } \\
\text { from } 8.1(7-9) \text { to } 4.6(3-7)\end{array}$ \\
\hline & & Male: 0\% & & & & & $\begin{array}{l}\text { VAS for overall satisfaction: } 6.8 \\
(5-8)\end{array}$ \\
\hline & & & & & & & $\begin{array}{l}\text { Satisfaction with procedure: } \\
\mathrm{n}=9(100)\end{array}$ \\
\hline & & & & & & & $\begin{array}{l}\text { Complications: } \\
\text { - Deep wound infection: } n=1(11 \%) \\
\text { - No loosening, screw failure, or } \\
\text { breakage } \\
\text { - No nonunion }\end{array}$ \\
\hline \multirow{7}{*}{ 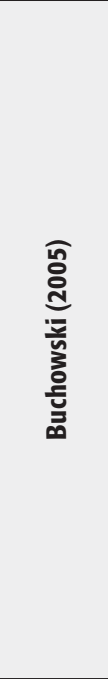 } & \multirow{7}{*}{ 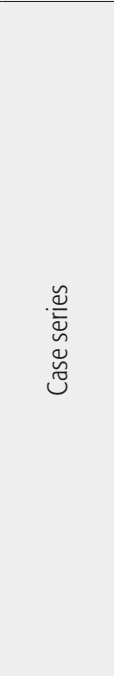 } & $\mathrm{N}=20$ patients & \multirow{4}{*}{$\begin{array}{l}\text { Failed conservative } \\
\text { therapy (physical therapy, } \\
\text { pain meds, lifestyle } \\
\text { modification): } 20 \text { (100) } \\
\text { Previous spine surgery: } \\
15(75)\end{array}$} & \multirow{7}{*}{$\begin{array}{l}\text { Pain relief with } \\
\text { intraarticular } \\
\text { sacroiliac joint } \\
\text { injections under } \\
\text { fluoroscopic } \\
\text { guidance }\end{array}$} & \multirow{7}{*}{$\begin{array}{l}\text { Used modified } \\
\text { Smith-Petersen } \\
\text { technique }\end{array}$} & \multirow{7}{*}{$\begin{array}{l}69 \text { months } \\
\text { (24-108) }\end{array}$} & Fusion rate: $n=17 / 20(85 \%)$ \\
\hline & & Unilateral (NR) & & & & & Revision surgery for nonunion: \\
\hline & & Bilateral (NR) & & & & & $n=3(15 \%)$ \\
\hline & & Age: 45.1 (21-66) & & & & & $\begin{array}{l}\text { Satisfaction with procedure: } \\
\mathrm{n}=12(60 \%)\end{array}$ \\
\hline & & Male: 15.0\% & \multirow[t]{3}{*}{$\begin{array}{l}\text { Previous fusion of sacrum: } \\
8(40)\end{array}$} & & & & $\begin{array}{l}\text { SF-36: statistically significant } \\
\text { improvement made in } 8 \text { of } 10 \text { outcome } \\
\text { categories }\end{array}$ \\
\hline & & & & & & & $\begin{array}{l}\text { AAOS modems instrument scores: } \\
\text { statistically significant improvement } \\
\text { found in satisfaction with symptoms, } \\
\text { neurogenic symptoms index, and pain/ } \\
\text { disability index }\end{array}$ \\
\hline & & & & & & & $\begin{array}{l}\text { Complications: } \\
\text { - Deep wound infection: } n=2(10 \%) \\
\text { - Pseudarthrosis: } n=3(15 \%) \\
\text { - Painful hardware: } n=1(5 \%)\end{array}$ \\
\hline \multirow{5}{*}{ 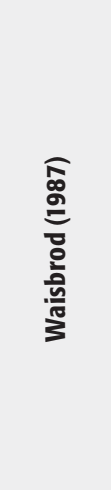 } & \multirow[b]{5}{*}{ 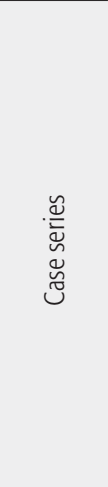 } & $\mathrm{N}=21$ patients & \multirow{5}{*}{$\begin{array}{l}\text { Previous operations for } \\
\text { complaints: } \\
\text { - discectomy: } 2 \text { (10) } \\
\text { - posterolateral spinal } \\
\text { fusion: } 5 \text { (24) } \\
\text { - bilateral total hip } \\
\text { replacement: } 2 \text { (10) }\end{array}$} & \multirow{5}{*}{$\begin{array}{l}\text { Injection of 10\% } \\
\text { NaCl solution } \\
\text { reproducing exact } \\
\text { pain pattern patient } \\
\text { complains of. Further } \\
\text { injection of local } \\
\text { anesthetic relieves } \\
\text { pain for duration of } \\
\text { drug's effect. }\end{array}$} & \multirow{5}{*}{$\begin{array}{l}\text { Articular surfaces } \\
\text { completely } \\
\text { excised. } \\
\text { Corticocancellous } \\
\text { bone graft taken } \\
\text { from iliac crest } \\
\text { and/or } \\
\text { tricalcium-phos- } \\
\text { phate ceramic } \\
\text { blocks interposed } \\
\text { under pressure } \\
\text { between } \\
\text { denuded } \\
\text { cancellous bone. }\end{array}$} & \multirow{5}{*}{$\begin{array}{l}30 \text { months } \\
(12-55)\end{array}$} & \multirow{5}{*}{$\begin{array}{l}\text { Satisfactory results (pain reduced } \\
\text { at least } 50 \%): n=11 / 22(50.0 \%) \\
\text { Nonunion: } n=2 / 22(9.1 \%) \\
\text { Complications: NR }\end{array}$} \\
\hline & & Unilateral $(n=20)$ & & & & & \\
\hline & & Bilateral $(n=1)$ & & & & & \\
\hline & & Age: 42.0 (20-58) & & & & & \\
\hline & & Male: $14.3 \%$ & & & & & \\
\hline
\end{tabular}

*VAS = visual analog scale

${ }^{\dagger} \mathrm{NR}=$ not reported 
Table 3 Characteristics of studies of denervation for sacroiliac joint pain

\begin{tabular}{|c|c|c|c|c|c|c|c|}
\hline $\begin{array}{l}\text { Author } \\
\text { (year) }\end{array}$ & $\begin{array}{l}\text { Study } \\
\text { design }\end{array}$ & $\begin{array}{l}\text { Study } \\
\text { population }\end{array}$ & $\begin{array}{l}\text { Previous } \\
\text { treatment }\end{array}$ & $\begin{array}{l}\text { Confirmed } \\
\text { diagnosis }\end{array}$ & $\begin{array}{l}\text { Denervation } \\
\text { description }\end{array}$ & $\begin{array}{l}\text { Follow-up } \\
\text { mean (range) }\end{array}$ & Outcomes \\
\hline \multirow{13}{*}{ 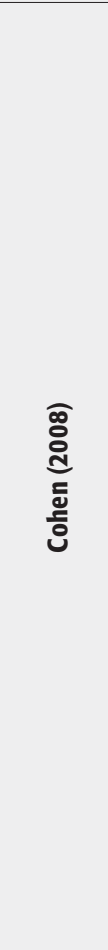 } & \multirow{13}{*}{ 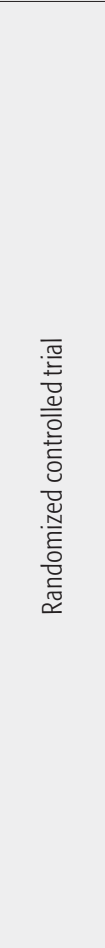 } & $\mathrm{N}=14$ patients & \multirow{8}{*}{$\begin{array}{l}\text { Physical therapy, } \\
\text { pharmacotherapy, } \\
\text { back surgery }\end{array}$} & \multirow{8}{*}{$\begin{array}{l}\text { Injections } \\
\text { containing } 2 \mathrm{~mL} \\
\text { of } 0.5 \% \\
\text { bupivacaine and } \\
1 \mathrm{~mL} \text { of } 40 \mathrm{mg} / \\
\mathrm{mL} \text { of } \\
\text { depo-methyl- } \\
\text { prednisolone }\end{array}$} & \multirow{8}{*}{$\begin{array}{l}\text { Cooled } \\
\text { radiofrequency (RF) } \\
\text { denervation of L4-5 } \\
\text { primary dorsal rami } \\
\text { and S1-3 lateral } \\
\text { branches performed } \\
\text { only after + 75\% } \\
\text { pain relief with L4-5 } \\
\text { and S1-3 lateral } \\
\text { branch blocks. }\end{array}$} & \multirow{8}{*}{$\begin{array}{l}6 \text { months } \\
\left(\mathrm{NR}^{*}\right)\end{array}$} & \multirow{2}{*}{$\begin{array}{l}\text { Numerical rating score (NRS) for pain: } \\
\text { improved average of } 3.5 \text { points from baseline } \\
(6.1 \pm 1.8) \text { to }(2.6 \pm 2.2)\end{array}$} \\
\hline & & Unilateral $(n=13)$ & & & & & \\
\hline & & Bilateral $(n=1)$ & & & & & \multirow{3}{*}{$\begin{array}{l}\text { Oswestry disability index: improved average } \\
\text { of } 14.5 \text { points from baseline }(37.1 \pm 10.6) \text { to } \\
(22.6 \pm 10.6)\end{array}$} \\
\hline & & Age: 51.9 (27-75) & & & & & \\
\hline & & \multirow[t]{2}{*}{ Male: $35.7 \%$} & & & & & \\
\hline & & & & & & & $\begin{array}{l}\text { Global perceived effect: 7/14 (50\%) at } 6 \\
\text { months }\end{array}$ \\
\hline & & \multirow{2}{*}{$\begin{array}{l}\mathrm{n}=14 \text { placebo } \\
\text { denervation }\end{array}$} & & & & & Medication reduction: $5 / 14(36 \%)$ at 6 months \\
\hline & & & & & & & $\begin{array}{l}\text { Complications: } \\
\text { - Only procedure-related pain and/or } \\
\text { temporary neuritis reported } \\
\text { - Transient non-painful buttock parasthesias } \\
(n=1)\end{array}$ \\
\hline & & \multirow{3}{*}{$\begin{array}{l}\mathrm{N}=11 / 14 \text { of } \\
\text { placebo patients } \\
\text { crossed over to } \\
\text { radiofrequency } \\
\text { denervation after } \\
\text { failed placebo } \\
\text { denervation at } 3 \\
\text { months }\end{array}$} & \multirow{5}{*}{$\begin{array}{l}\text { Physical therapy, } \\
\text { pharmacotherapy, } \\
\text { back surgery, } \\
\text { placebo } \\
\text { denervation }\end{array}$} & \multirow{5}{*}{$\begin{array}{l}\text { Injections } \\
\text { containing } 2 \mathrm{~mL} \\
\text { of } 0.5 \% \\
\text { bupivacaine and } \\
1 \mathrm{~mL} \text { of } 40 \mathrm{mg} / \\
\mathrm{mL} \text { of } \\
\text { depo-methyl- } \\
\text { prednisolone }\end{array}$} & \multirow{5}{*}{$\begin{array}{l}\text { Conventional } \\
\text { (non-cooled) RF } \\
\text { denervation of L4-5 } \\
\text { primary dorsal rami } \\
\text { and S1-3 lateral } \\
\text { branches performed } \\
\text { only after failed } \\
\text { placebo denervation }\end{array}$} & \multirow{5}{*}{$\begin{array}{l}6 \text { months } \\
\text { (NR) }\end{array}$} & $\begin{array}{l}\text { NRS for pain: improved average of } 3.4 \text { points } \\
\text { from baseline }(6.5 \pm 1.9) \text { to } 6 \text { months } \\
\text { post-denervation }(3.1 \pm 2.1)\end{array}$ \\
\hline & & & & & & & $\begin{array}{l}\text { Oswestry disability index: improved average } \\
\text { of } 23.6 \text { points from baseline }(47.9 \pm 9.3) \text { to } 6 \\
\text { months post denervation }(24.3 \pm 21.0)\end{array}$ \\
\hline & & & & & & & Global perceived effect: $5 / 11(46 \%)$ at 6 months \\
\hline & & Age: $51.8(31-74)$ & & & & & Medication reduction: $3 / 11$ (27\%) at 6 months \\
\hline & & Male: $42.8 \%$ & & & & & \\
\hline \multirow{5}{*}{ 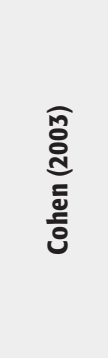 } & \multirow{5}{*}{ 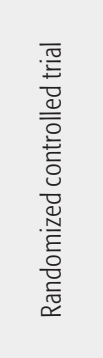 } & $\mathrm{N}=9$ patients & \multirow{5}{*}{$\begin{array}{l}\text { Physical therapy, } \\
\text { previous blocks, } \\
\text { back surgery, pain } \\
\text { medicine } \\
\text { management }\end{array}$} & \multirow{5}{*}{$\begin{array}{l}\text { Injections } \\
\text { performed with } \\
80 \mathrm{mg} \\
\text { triamcinolone } \\
\text { acetonide } \\
\text { steroid and } 1 \text { to } \\
3 \mathrm{~mL} 0.5 \% \\
\text { ropivacaine or } \\
0.5 \% \\
\text { bupivacaine }\end{array}$} & \multirow{5}{*}{$\begin{array}{l}\text { RF denervation of } \\
\text { L4-5 primary dorsal } \\
\text { rami and S1-3 lateral } \\
\text { branches performed } \\
\text { only after }>50 \% \text { pain } \\
\text { relief with L4-5 and } \\
\text { S1-3 lateral branch } \\
\text { blocks }\end{array}$} & \multirow{5}{*}{$\begin{array}{l}9 \text { months } \\
\text { (NR) }\end{array}$} & \multirow{5}{*}{$\begin{array}{l}\text { Visual analog scale (VAS) for pain: } \\
\text { improved average of } 4.1 \text { points from baseline } \\
\text { (6.3) to } 9 \text { months post denervation ( } 2.2) \\
\text { Complications: } \\
\text { No complications with any of the procedures }\end{array}$} \\
\hline & & Unilateral (NR) & & & & & \\
\hline & & Bilateral (NR) & & & & & \\
\hline & & Age: $50.0(31-81)$ & & & & & \\
\hline & & Male: $53.9 \%$ & & & & & \\
\hline \multirow[b]{5}{*}{ 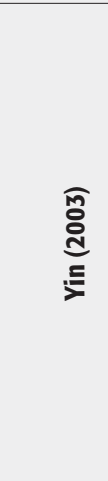 } & \multirow[b]{5}{*}{ 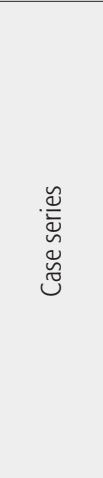 } & $\mathrm{N}=14$ patients & \multirow{5}{*}{$\begin{array}{l}\text { Physical therapy, } \\
\text { medical therapy, } \\
\text { manual therapy, } \\
\text { home-based } \\
\text { flexibility and } \\
\text { exercise programs, } \\
\text { TENS, anesthetic } \\
\text { injections, back } \\
\text { surgery (lumbar } \\
\text { discectomy, fusion } \\
\text { or laminectomy) }\end{array}$} & \multirow{5}{*}{$\begin{array}{l}\text { Two separate } \\
\text { deep } \\
\text { interosseous } \\
\text { ligamentous } \\
\text { injections with } 5 \\
\mathrm{~mL} \text { of } 0.5 \% \\
\text { bupivacaine } \\
\text { containing } 4 \\
\mathrm{mg} / \mathrm{mL} \text { of } \\
\text { triamcinolone }\end{array}$} & \multirow{5}{*}{$\begin{array}{l}\text { Sensory } \\
\text { stimulation-guided } \\
\text { RF denervation of L5 } \\
\text { posterior sensory } \\
\text { branch and the } \\
\text { lateral branches of } \\
\text { S1-3 }\end{array}$} & \multirow{5}{*}{$\begin{array}{l}6 \text { months } \\
\text { (NR) }\end{array}$} & \multirow{5}{*}{$\begin{array}{l}\text { Visual integer pain score (VIPS): 64\% } \\
\text { successful outcome (defined as 60\% } \\
\text { consistent subjective relief and greater than a } \\
50 \% \text { consistent decrease in VIPS, maintained } \\
\text { for at least } 6 \text { months) } \\
\text { Complications: } \\
\text { - No identifiable surgical complications } \\
\text { - Cutaneous numbness over one buttock } \\
\text { lasting } 6 \text { months ( } \mathrm{n}=1 \text { ) } \\
\text { - Most patients with transient buttock } \\
\text { cutaneous dysesthesia } \\
\text { - No bowel or bladder dysfunction } \\
\text { - No postprocedure infections }\end{array}$} \\
\hline & & Unilateral (NR) & & & & & \\
\hline & & Bilateral (NR) & & & & & \\
\hline & & Age: 53.7 (35-79) & & & & & \\
\hline & & Male: $28.6 \%$ & & & & & \\
\hline \multirow{6}{*}{ 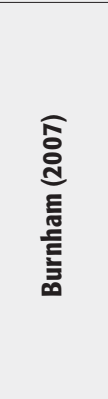 } & & $\mathrm{N}=9$ patients & Back exercises, & Local anesthetic & RF denervation of & 12 months & Patient satisfaction: $8 / 9(89 \%)$ \\
\hline & & Unilateral $(n=8)$ & $\begin{array}{l}\text { manual therapy, } \\
\text { physical therapy, }\end{array}$ & joint block & $\begin{array}{l}\text { posterior sensory } \\
\text { nerves }\end{array}$ & (NR) & NRS for pain: improved average of 3.5 points \\
\hline & & Bilateral $(n=1)$ & medical therapy, & & & & $\begin{array}{l}\text { from baseline }(\text { mean }=8) \text { to } 12 \text { months } \\
\text { post-denervation (mean }=4.5)\end{array}$ \\
\hline & 呟 & & & & & & Revised Oswestry disability index: improved \\
\hline & $\stackrel{心}{心}$ & Male: $22.2 \%$ & & & & & $\begin{array}{l}\text { average of } 18 \text { points from baseline }(\text { mean }=56) \\
\text { to } 12 \text { months postdenervation (mean }=38)\end{array}$ \\
\hline & & & & & & & $\begin{array}{l}\text { Complications: } \\
\text {-Numbness and itchiness of skin overlying } \\
\text { treated sacroiliac joint }(n=3)\end{array}$ \\
\hline
\end{tabular}




\begin{tabular}{|c|c|c|c|c|c|c|c|}
\hline $\begin{array}{l}\text { Author } \\
\text { (year) }\end{array}$ & $\begin{array}{l}\text { Study } \\
\text { design }\end{array}$ & $\begin{array}{l}\text { Study } \\
\text { population }\end{array}$ & $\begin{array}{l}\text { Previous } \\
\text { treatment }\end{array}$ & $\begin{array}{l}\text { Confirmed } \\
\text { diagnosis }\end{array}$ & $\begin{array}{l}\text { Denervation } \\
\text { description }\end{array}$ & $\begin{array}{l}\text { Follow-up } \\
\text { mean (range) }\end{array}$ & Outcomes \\
\hline \multirow{7}{*}{ 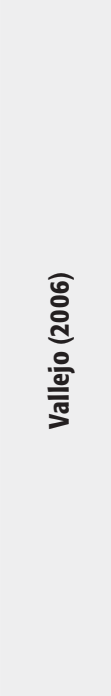 } & \multirow{7}{*}{ 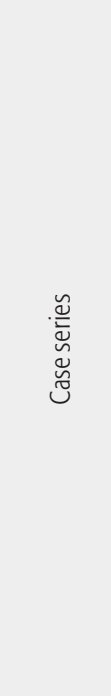 } & $\mathrm{N}=22$ patients & \multirow{7}{*}{$\begin{array}{l}\text { Physical therapy, } \\
\text { medical therapy }\end{array}$} & \multirow{7}{*}{$\begin{array}{l}\text { Two or more } \\
\text { fluoroscopically } \\
\text { guided } \\
\text { injections of } \\
\text { corticosteroid/ } \\
\text { local anesthetic }\end{array}$} & \multirow{7}{*}{$\begin{array}{l}\text { Pulsed RF } \\
\text { denervation of } \\
\text { medial branch of } L 4 \text {, } \\
\text { posterior rami of } L 5 \text {, } \\
\text { and the lateral } \\
\text { branches of S1-2. }\end{array}$} & \multirow{7}{*}{$\begin{array}{l}6 \text { months } \\
\text { (NR) }\end{array}$} & \multirow{7}{*}{$\begin{array}{l}\text { VAS pain score: improved average of } 4.9 \\
\text { points from baseline }(7.6 \pm 1.7) \text { to } 6 \text { months } \\
\text { postdenervation }(2.7 \pm 1.8)(P<.0001) \\
\text { FACIT quality of life scores: } \\
\text { - Physical well-being: baseline }(1.6 \pm 0.7) \text { and } \\
6 \text { months post-denervation }(1.1 \pm 0.5) \\
(P<.0001) \\
\text { - Social-well being: baseline }(3.1 \pm 0.9) \text { and } 6 \\
\text { months post-denervation }(3.2 \pm 0.9) \\
(P=.039) \\
\text { - Emotional well-being: baseline }(1.3 \pm 0.5) \text { and } \\
6 \text { months post-denervation }(1.0 \pm 0.4) \\
(P=.014) \\
\text { - Functional well-being: baseline }(1.4 \pm 0.8) \text { and } \\
6 \text { months post-denervation }(2.1 \pm 1.0) \\
\text { ( } P<.0001) \\
\text { Complications: } \\
\text { No complications directly or indirectly related } \\
\text { to procedure }\end{array}$} \\
\hline & & & & & & & \\
\hline & & Bilateral (NR) & & & & & \\
\hline & & Age: $55.9( \pm 14)$ & & & & & \\
\hline & & Male: $18.2 \%$ & & & & & \\
\hline & & & & & & & \\
\hline & & & & & & & \\
\hline
\end{tabular}

\section{EVIDENCE SUMMARY}

Question 1: To compare the effectiveness of fusion versus denervation for chronic sacroiliac joint pain after failed conservative management

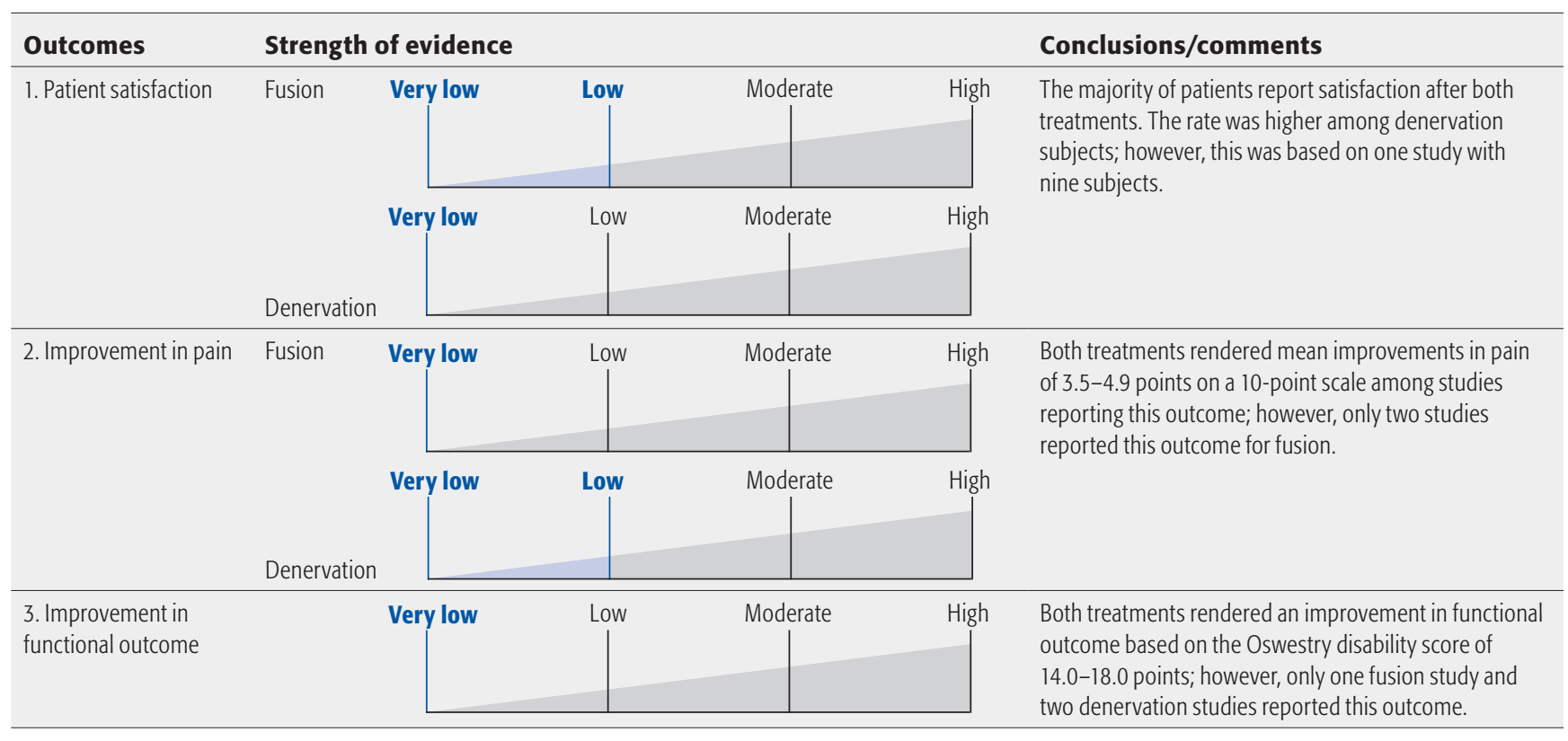

Question 2: To compare the safety of fusion versus denervation for chronic sacroiliac joint pain after failed conservative management

\begin{tabular}{|c|c|c|c|c|c|}
\hline \multirow[t]{3}{*}{ 4. Complications } & \multirow[t]{3}{*}{ Very low } & \multirow{3}{*}{ Low } & \multirow{3}{*}{ Moderate } & \multirow{3}{*}{ High } & \multirow{3}{*}{$\begin{array}{l}\text { Rates of complications were higher among fusion studies } \\
(13.7 \%) \text { compared to denervation studies }(7.3 \%) \text {; } \\
\text { however, it is important to note that these are different } \\
\text { study populations and the follow-up times are much } \\
\text { shorter among denervation studies. }\end{array}$} \\
\hline & & & & & \\
\hline & & & & & \\
\hline 5. Infections & Very low & Low & Moderate & High & $\begin{array}{l}\text { Only fusion studies reported infections ( } 5.3 \%) \text {. No } \\
\text { infections were reported among denervation patients. }\end{array}$ \\
\hline
\end{tabular}




\section{DISCUSSION}

- The majority of subjects report satisfaction after either treatment. Both treatments appear to demonstrate improvement in outcomes from pre to posttreatment during their follow-up period.

- Complication rates and infection rates are higher among those undergoing fusion compared to those undergoing denervation.

- The existing literature is limited to case series. No studies were identified that compared treatments in the same patient population. Given these limitations, pooled rates from these studies must be taken with caution. The open fusion studies reported poorer results and higher complication rates than the percutaneous studies. However, the concept of 'fusion' of the sacroiliac joint after percutaneous fixation with hollow screws or cages filled with bone-graft substitute was based on the absence of loosening on plain x-rays or confirmation of trabecular continuity across the implants on CT scanning. Also, many of the patients in the fusion series had undergone previous spinal surgery, suggesting that a positive response to sacroiliac blocks does not predict successful pain relief after sacroiliac fusion in patients with chronic pain syndromes.

- All of the denervation studies have short follow-up periods, raising the question of duration of effect given that many reported studies of lumbar facet joint denervation show loss of efficacy after about 2 years.

- There is a clear need for more properly constructed comparative studies to establish whether chronic sacroiliac joint pain can be better managed with invasive pain relieving techniques than conventional conservative therapies.

\section{REFERENCES}

1. Wise CL, Dall BE (2008) Minimally invasive sacroiliac arthrodesis: outcomes of a new technique. J Spinal Disord Tech; 21(8):579-584.

2. Schutz U, Grob D (2006) Poor outcome following bilateral sacroiliac joint fusion for degenerative sacroiliac joint syndrome. Acta Orthop Belg; 72:296-308.

3. Al-Khayer A, Hegarty J, Hahn D, et al (2008) Percutaneous sacroiliac joint arthrodesis: a novel technique. J Spinal Disord Tech; 21(5):359-363.

4. Buchowski JM, Kebaish KM, Sinkov V, et al (2005) Functional and radiographic outcome of sacroiliac arthrodesis for the disorders of the sacroiliac joint. Spine J; 5(5):520-528; discussion 529.

5. Burnham RS, Yasui Y (2007) An alternate method of radiofrequency neurotomy of the sacroiliac joint: a pilot study of the effect on pain, function, and satisfaction. Reg Anesth Pain Med; 32(1):12-19.

6. Cohen SP, Hurley RW, Buckenmaier CC 3rd, et al (2008) Randomized placebo-controlled study evaluating lateral branch radiofrequency denervation for sacroiliac joint pain. Anesthesiology; 109(2):279-288.

7. Cohen SP, Abdi S (2003) Lateral branch blocks as a treatment for sacroiliac joint pain: a pilot study. Reg Anesth Pain Med; 28(2):113-119.

8. Vallejo R, Benyamin RM, Kramer J, et al (2006) Pulsed radiofrequency denervation for the treatment of sacroiliac joint syndrome. Pain Med; 7(5):429-434.

9. Khurana A, Guha AR, Mohanty $K$, et al (2009) Percutaneous fusion of the sacroiliac joint with hollow modular anchorage screws: clinical and radiological outcome. J Bone Joint Surg Br; 91(5):627-631.

10. Yin W, Willard F, Carreiro J, et al (2003) Sensory stimulation-guided sacroiliac joint radiofrequency neurotomy: technique based on neuroanatomy of the dorsal sacral plexus. Spine; 28(20):2419-2425.

11. Waisbrod H, Krainick JU, Gerbershagen HU (1987) Sacroiliac joint arthrodesis for chronic lower back pain. Arch Orthop Trauma Surg; 106(4):238-240. 


\section{EDITORIAL STAFF PERSPECTIVE}

Our reviewers were unanimously in favor of publication of this review, despite the controversial nature of a number of aspects of this condition-starting with uncertainty surrounding physical examination, continuing with diagnostic confirmation and finally treatment. The results of this systematic review point out a commonly encountered conundrum: while there seems to be some evidence suggesting a 'positive treatment effect' as reported by patients, closer inspection of the data at hand produces more questions than answers. In summary, the role of the sacro-iliac joint in low back pain and its management remain very much unclear to the present date and is, perhaps, somewhat overemphasized by some. Identification of pathologic entities such as presented by the authors-clear instability, severe degeneration, infection, neoplasia-remains a helpful contribution to patient care by an informed practitioner. Should surgical stabilization be chosen for a sufferer of SI-joint pain, the current state of the literature unfortunately allows little or no real insights due to unclear handling of several confounding variables (ie, previous spine fusion, osteoporosis, obesity, pelvic alignment, physical fitness levels) and inconsistent definition of desired surgical outcomes - are true fusions actually achieved in a large number of the described techniques? That said, are solid fusions actually necessary for a satisfactory outcome? Do the outcomes justify the means? Many questions, few real answers, much to do in the future. 